\title{
Isolation and Characterization of Nicotine Reducing Probiotics
}

\author{
Bhavika Chauhan and Nileshkumar Pandya* \\ Department of Microbiology, Arts, Science \& Commerce College, Kamrej Cross Road, Surat, \\ Gujarat-394185, India \\ *Corresponding author
}

\begin{abstract}
A B S T R A C T
Keywords

Nicotine,

Lactobacillus

sporogenes,

Lactobacillus casei,

nicotine reducing

activity, Probiotic

Article Info

Accepted:

10 June 2019

Available Online:

10 July 2019

A total of seven microorganisms were isolated from different probiotic sources. The nicotine reducing activity of isolates was tested by general analytical techniques. Amongst them isolates BC6 and BC7 possess maximum nicotine reducing activity therefore it was selected for further investigation. Both isolates could utilize up to $1 \mathrm{~g} / \mathrm{l}$ nicotine as the sole source of carbon and nitrogen, and optimum nicotine reducing activity obtained at $\mathrm{pH} 7$ by inoculating with inoculum size of 1 McFarland standard units. After morphological and biochemical characterization and according to information given on leaflet of probiotic product, isolate BC6 and BC7 were identified as Lactobacillus sporogenes and Lactobacillus casei, respectively.
\end{abstract}

\section{Introduction}

Nicotine, 3-(1-methyl-2-pyrrolidinyl) pyridine is an alkaloid found in the nightshade Solanaceae family of plants, predominantly in the leaves of tobacco and it is a colorless, less to pale yellow, hygroscopic oily liquid (Hossain and Salehuddin, 2013). People can smoke, chew or sniff tobacco. Smoked tobacco products include cigarettes, cigars, bidis and kretes and chewed tobacco products include chewing tobacco, snuff, dip and snus; snuff can also be sniffed (NIDA, 2018). Nicotine is an addictive substance that can lead to nicotine dependence and addictive behaviour in human populations (Niu et al.,
2000). Nicotine can cause cancer, gene mutation \& malformation (Yildiz, 2004) and neurotoxin developmental effects of nicotine can naturally affect a variety of cellular processes such as generation of oxidative radicals, apoptosis and hyperplasia of cell, enhancing gene expression to secrete hormones and regulation of enzymatic activity (Yildiz, 2004; Yildiz et al., 1998; Qiao et al., 2005 and Gurusamy and Natarajan, 2013).

A number of microorganisms able to degrade nicotine have been isolated and characterized till now, including bacteria, actinomycetes and fungi (Brandsch, 2006; Li et al., 2010; Meng et al., 2010; Wang et al., 2009 \& Li et al., 
2014). No one is isolated and characterized probiotic for removal of nicotine on human beings yet. Tobacco waste degrading bacteria can't directly use on human as it may be adversely affect human beings. The intention of this study is to isolate and characterise probiotics that can reduce nicotine concentration in the human body.

\section{Materials and Methods}

\section{Isolation of probiotics}

For isolation of probiotics, various probiotic sources such as Curd, Vitagut probiotic capsule and Yakult fermented milk drink were streaked on sterile nutrient agar plate and sterile MRS agar plate and incubated at $37{ }^{\circ} \mathrm{C}$ for $48 \mathrm{hrs}$. After incubation developed colonies were transferred and streaked on the new agar plates repeatedly until a pure isolate was obtained. Isolates were transferred individually on sterile nutrient agar slant and preserved at $4^{\circ} \mathrm{C}$ for further use.

Enrichment culture and isolation and identification of nicotine reducing probiotics

Probiotics isolated as above were enriched for determining their nicotine reducing activity. The isolates were added into sterile liquid nicotine medium (NIM) containing $\mathrm{Na}_{2} \mathrm{HPO}_{4}$ $6 \mathrm{~g} / \mathrm{l}, \mathrm{KH}_{2} \mathrm{PO}_{4} 3 \mathrm{~g} / \mathrm{l}, \mathrm{NH}_{4} \mathrm{Cl} 1 \mathrm{~g} / \mathrm{l}, \mathrm{NaCl} 0.5 \mathrm{~g} / \mathrm{l}$, $\mathrm{MgSO}_{4} 0.12 \mathrm{~g} / \mathrm{l}, \mathrm{CaCl}_{2} 0.1 \mathrm{~g} / \mathrm{l}$ and nicotine 0.2 $\mathrm{g} / \mathrm{l}$, (Wei et al., 2008) in which nicotine (extracted from tobacco; Singh et al., 2013) was the sole source of carbon and nitrogen, nicotine was sterilized separately by filtration and added into media before inoculation and broth were inoculated with isolates and incubated at $37{ }^{\circ} \mathrm{C}$. After 4 days, the culture was used as inoculum and streaked onto the solid nicotine medium plates containing 0.2 $\mathrm{g} / \mathrm{l}$ nicotine. After incubation at $37^{\circ} \mathrm{C}$ for 72 hrs, colonies began to appear on the plates and these isolates were selected for this study.
Selected isolates were morphologically and biochemically characterized by Gram's staining and various biochemical test as per standard methods (Cappuccino and Sherman, 1992).

\section{Screening of microorganisms for nicotine reducing activity}

Different isolates obtained on NIM agar plates were screened for their nicotine reducing activity on sterile NIM broth containing $0.5 \mathrm{~g} / \mathrm{l}$ nicotine. Isolates were inoculated in sterile NIM broth and incubated at $37{ }^{\circ} \mathrm{C}$ and determined cell density by taking absorbance at 600nm (Liu et al., 2014) and nicotine reducing activity at $1 \mathrm{hr}$ intervals. Uninoculated NIM broth containing $0.5 \mathrm{~g} / \mathrm{l}$ nicotine was used as control.

\section{Analytical method for determining nicotine reducing activity of isolates}

\section{Spectrophotometric method}

At predetermined time intervals, the samples of inoculated broth were taken and cells were removed by centrifugation at $10,000 \mathrm{rpm}$ for $10 \mathrm{~min} .1 \mathrm{ml}$ of the supernatant was mixed with $0.5 \mathrm{ml}$ of bromine water and shaken it for 2 min. By the drop wise addition of formic acid, the excess of bromine was removed then $1 \mathrm{ml}$ each of potassium iodide and leuco crystal violet was added and shaken for few minutes. $1 \mathrm{ml}$ of $2 \mathrm{M}$ sodium hydroxide was added drop wise and made final volume to the $25 \mathrm{ml}$ with double distilled water and the absorbance was measured at $592 \mathrm{~nm}$ after 25 min (Asthana et al., 2004).

\section{Thin Layer Chromatography (TLC)}

After 6 hours of nicotine broth inoculation with isolates, the samples were centrifuged and supernatant was used for TLC analysis. On silica gel $60 \mathrm{~F}_{254}$ TLC aluminium sheets, nicotine reducing activity of microbes were 
determined by using Toluene - Isopropanol Ammonia (100:25:1) as a mobile phase and spraying with Dragendroff's reagent (Svendsen et al., 2011).

Optimal conditions for growth and nicotine reducing activity of isolates

Potent microbes were selected by primary screening and its maximum nicotine reducing activity was determined by inoculating them in sterile NIM broth containing nicotine concentration ranging from 0.5 to $1.5 \mathrm{~g} / \mathrm{l}$ and determined cell density and nicotine reducing activity as predetermined methods. Uninoculated nicotine medium were used as control. The effect of $\mathrm{pH}$ on isolates was tested by adjusting a variety of $\mathrm{pH}$ condition of broth with $1 \mathrm{M}$ of $\mathrm{HCl}$ or $\mathrm{NaOH}$ to $\mathrm{pH} 6$ to 8 , respectively. For determining the effect of inoculum size on nicotine reducing activity, the nicotine broth was inoculated with various inoculum sizes adjusted to 0.5, 1.0 and 2.0 McFarland Standard units and analysed their nicotine reducing activity (McFarland J., 1907). All experiments were performed in triplicate.

\section{Results and Discussion}

\section{Isolation of probiotics}

Total 8 isolates were obtained on Nutrient agar and MRS agar plates, amongst them 4, 3 and 1 from curd, vitagut capsule and yakult fermented milk drink, respectively.

Enrichment culture and isolation and identification of nicotine reducing probiotics

After 4 days of Isolate addition into liquid nicotine medium, turbidity observed in broth indicating their growth and their colony formation on NIM agar plate containing nicotine as sole source of carbon and nitrogen after $72 \mathrm{hrs}$ indicating they possessed nicotine reducing activity.

All isolates able to form visible colony on the nicotine agar plate except one isolated from curd showing that it was incompetent to reduce nicotine concentration.

The colony morphology of isolate BC6 was large, round, moist, opaque, raised and entire edge. It was a Gram-positive and long rod shaped bacterium. The colony morphology of isolate BC7 was large, round, mucoid, opaque, flat \& entire edge. It was a Gram-positive and short rod shaped bacterium. Isolate BC6 and BC7 showing maximum nicotine reducing activity were selected for this investigation, which were identified as Lactobacillus sporogenes and Lactobacillus casei and obtained from vitagut capsule and yakult fermented milk drink, respectively. They are identified from morphological and biochemical characterization and according to information given on leaflet of probiotic product.

\section{Screening of microorganisms for nicotine reducing activity}

A seven isolates showing colony formation on the NIM agar plate and their nicotine reducing activity into NIM broth containing $0.5 \mathrm{~g} / \mathrm{l}$ nicotine was determined by two methods.

\section{Analytical method for determining nicotine reducing activity of isolates}

\section{Spectrophotometric method}

The cell growth and nicotine reducing activity of isolates into nicotine medium was determined by taking optical density of broth at $600 \mathrm{~nm}$ and at 592nm as above described method, respectively. 
A

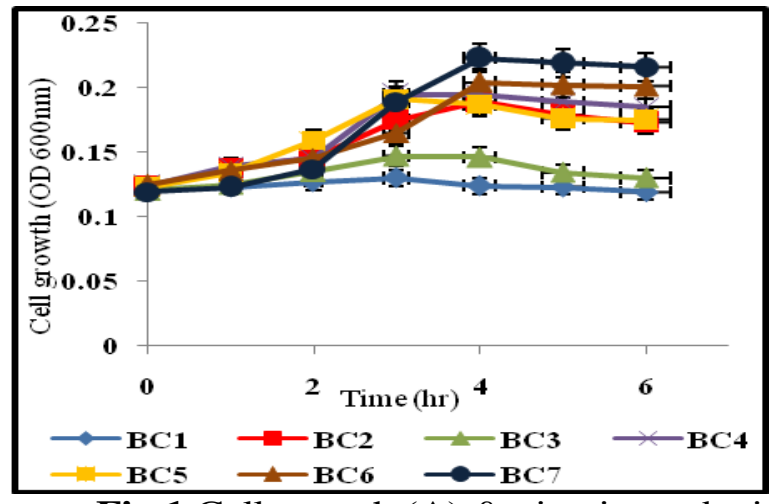

B

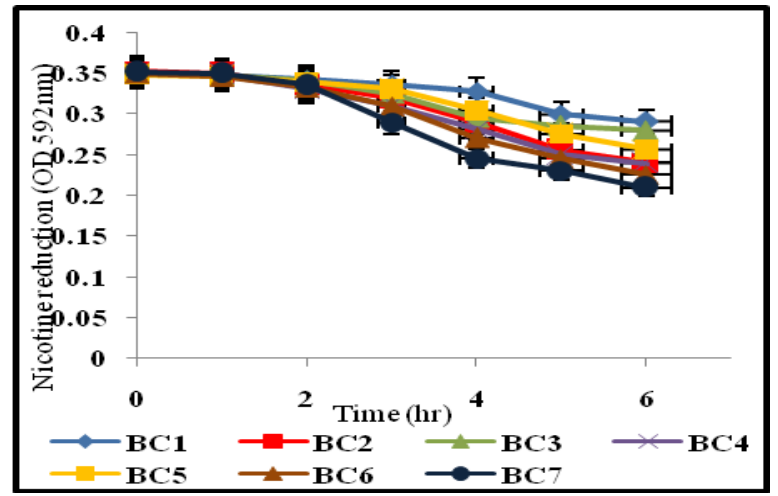

Fig.1 Cell growth (A) \& nicotine reducing activity (B) of isolates in $0.5 \mathrm{~g} / \mathrm{l}$ nicotine Containing medium

A

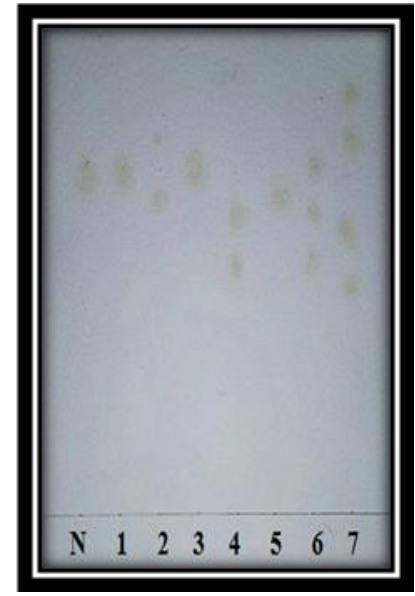

B

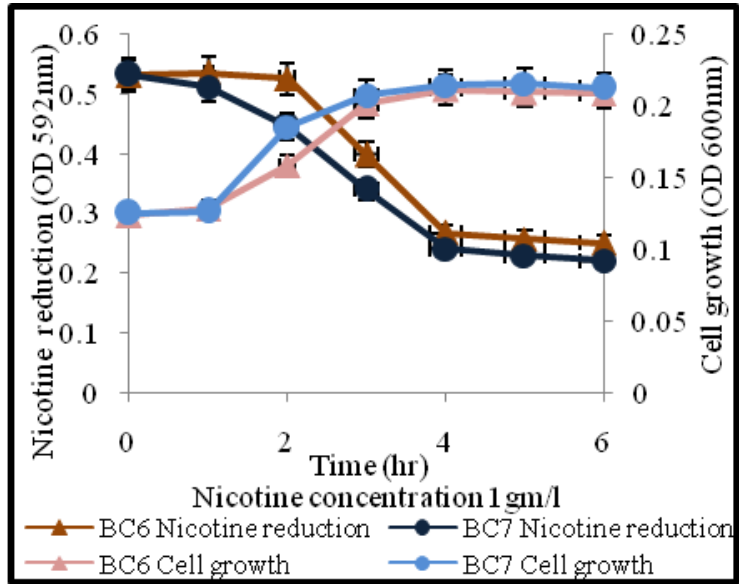

Fig.2 TLC analysis (A), cell growth and nicotine reducing activity of BC6 and BC7 in 1 g/l nicotine containing medium (B)

$\mathbf{A}$

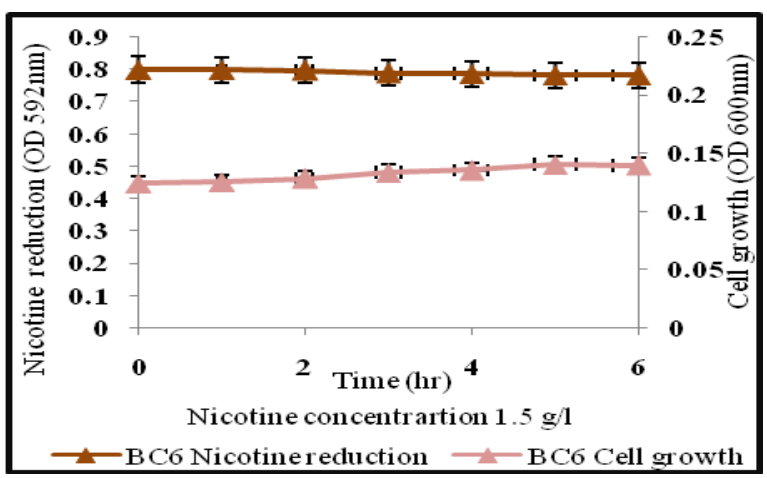

B

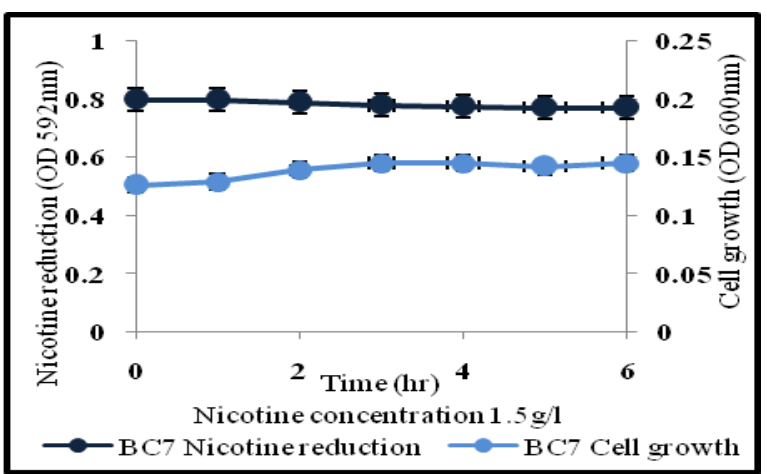

Fig.3 Cell growth and nicotine reducing activity of BC6 (A) \& BC7 (B) in $1.5 \mathrm{~g} / 1$ nicotine containing medium 
A

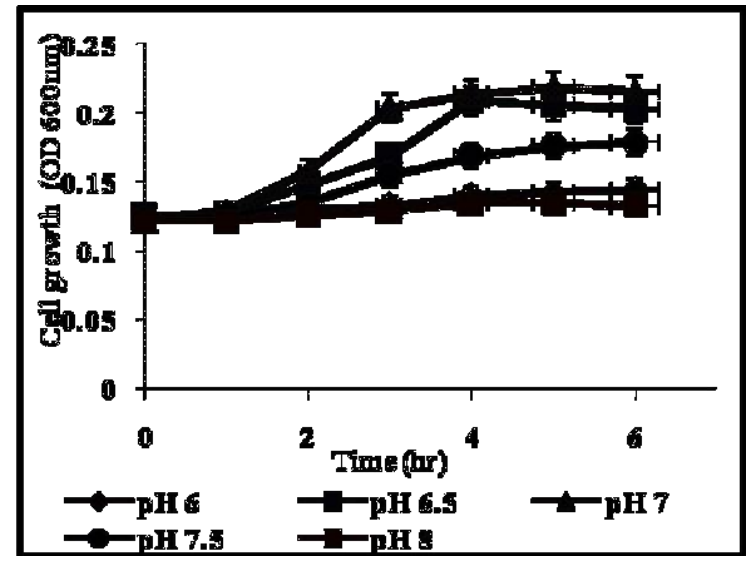

C

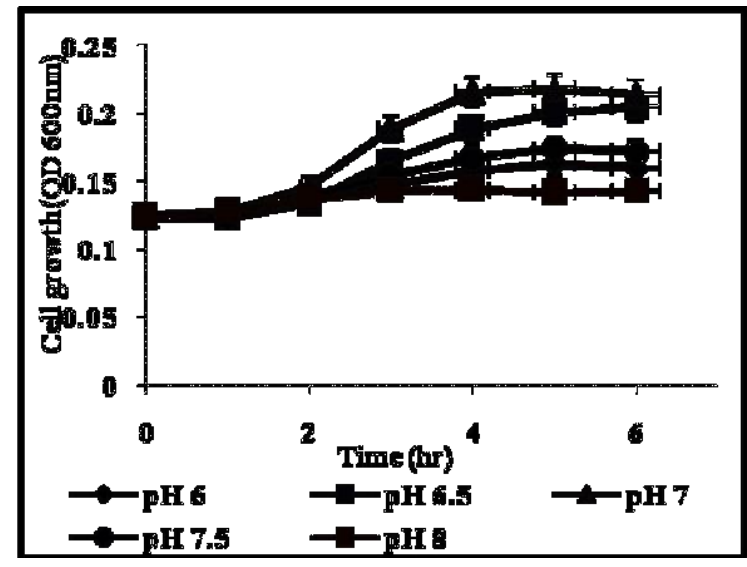

$\mathbf{E}$

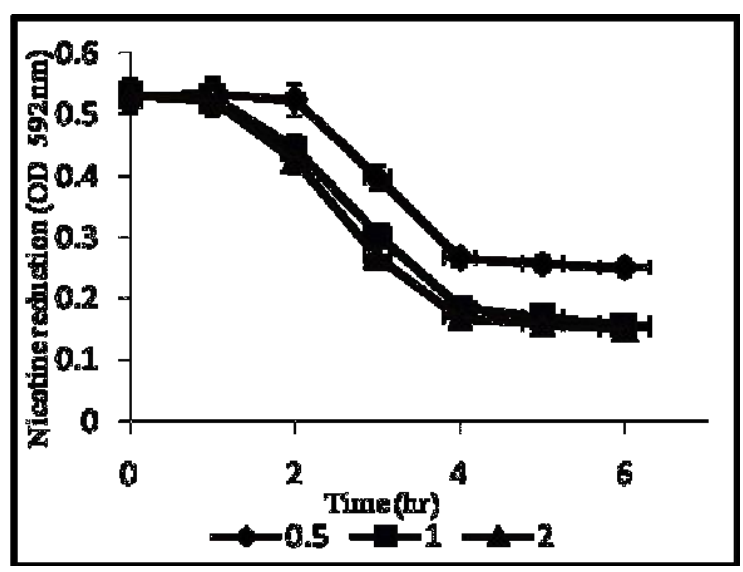

B

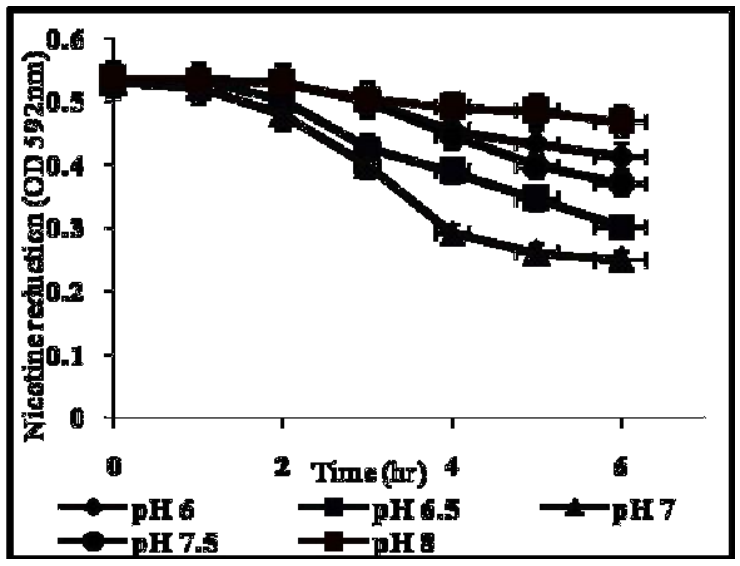

D

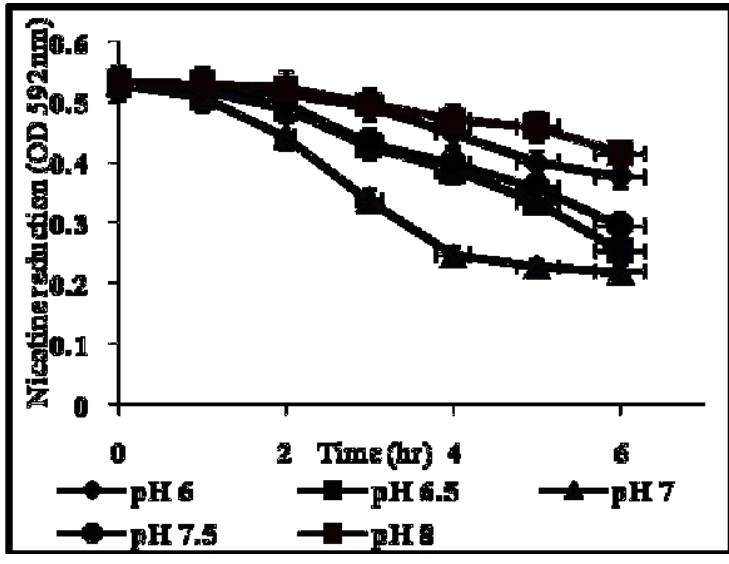

$\mathbf{F}$

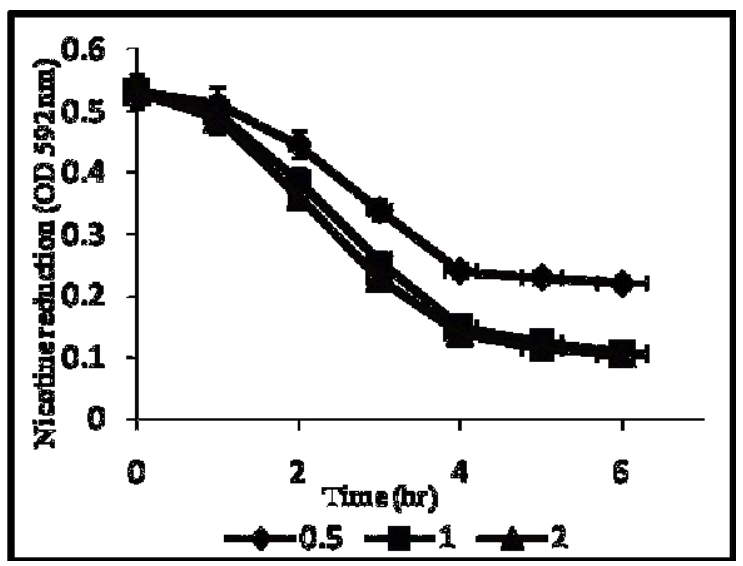

Fig.4 Effect of pH on cell growth of BC6 (A) \& BC7 (C) and nicotine reducing activity of BC6 (B) \& BC7 (D), Effect of inoculum size imicotine reducing activity of BC6(E) $\& \mathrm{BC} 7(\mathrm{~F})$ 
As shown in figure 1(A), the growth of isolate BC6 and BC7 was observed maximum in compare to other. The depletion of absorbance of nicotine medium at $592 \mathrm{~nm}$ was suggested that nicotine concentration decreased in the broth.

A minimal optical density at $592 \mathrm{~nm}$ of nicotine medium inoculated with strain BC6 and $\mathrm{BC} 7$ after $6 \mathrm{hrs}$ was manifested that their maximum nicotine reducing activity in compare to other isolates (Figure 1B).

\section{Thin layer chromatography}

After $6 \mathrm{hrs}$ of incubation of isolates in $0.5 \mathrm{~g} / \mathrm{l}$ nicotine containing medium, the result of TLC analysis were shown in figure 2(A) in which $\mathrm{N}$ indicated nicotine broth without microbes inoculation as control and nicotine broth inoculated with $\mathrm{BC} 1, \mathrm{BC} 2, \mathrm{BC} 3, \mathrm{BC} 4$, $\mathrm{BC} 5, \mathrm{BC} 6$ and $\mathrm{BC} 7$ were labelled as 1 to 7 , respectively. In comparison to control, more spots observed with BC6 and BC7 indicates the probable nicotine destruction to more than one product.

\section{Optimal conditions for growth and nicotine reducing activity of isolates}

The effects of nicotine concentration on cell growth and nicotine reducing activity of two potent isolates were investigated at nicotine concentration ranging from 0.5-1.5 g/l. As shown in figure $2(\mathrm{~B})$, the maximum cell growth and nicotine reducing activity of both isolates was observed at $1 \mathrm{~g} / \mathrm{l}$ nicotine concentration and both isolates were unable to grow on $1.5 \mathrm{~g} / \mathrm{l}$ nicotine containing medium so that nicotine reducing activity was not visible at this concentration. The obtained results are similar to the work done by Liu et al., 2014, where the optimal growth of Pseudomonas geniculate N1 was observed at $1 \mathrm{~g} / \mathrm{l}$ nicotine in which nicotine was the sole source of carbon and nitrogen.
A cell growth and nicotine reducing activity of isolates with nicotine was observed from $\mathrm{pH} 6$ to 8. The maximum growth and nicotine reducing activity of both isolates on nicotine medium was at $\mathrm{pH} \mathrm{7,} \mathrm{which} \mathrm{suggest} \mathrm{that} \mathrm{they}$ prefers a neutral environment for reduction of nicotine concentration whereas the optimum growth of Acinetobacter sp. ND12 with nicotine was observed between $\mathrm{pH} 4$ and $\mathrm{pH}$ 6 (Li et al., 2011). In this study isolated bacteria showed optimal activity close to neutral $\mathrm{pH}$ and saliva $\mathrm{pH}$ also normally nearby it (Baliga et al., 2013) so these strains may be helpful to reduce nicotine concentration from smokeless tobacco in mouth (Fig. 3).

The influence of inoculum size on cell growth and nicotine reducing activity were determined at different inoculum size ranged from 0.5 to 2 McFarland standard units. Figure 4(E) and 4(F) clearly indicated that the nicotine reducing activity of microorganisms improved as the inoculum size was increased. The nicotine broth inoculated with 2 McFarland standard unit inoculum size containing culture was shown the absorbance at $592 \mathrm{~nm}$ nearer to develop by $1 \mathrm{McFarland}$ standard unit inoculum size containing culture of both isolates it suggest that the effect of inoculum size above 1 McFarland standard unit on nicotine reducing activity was become a stagnant.

Isolates $\mathrm{BC} 6$ and $\mathrm{BC} 7$ possess maximum nicotine reducing activity, they could utilize nicotine up to $1 \mathrm{~g} / \mathrm{l}$ as the sole source of carbon and nitrogen. Optimum nicotine reducing activity obtained at $\mathrm{pH} \quad 7$ by inoculating the isolates with inoculum size of $1 \mathrm{McF}$ arland standard units.

\section{References}

Asthana, A., Rastogi, R., Sunita, G., and Gupta, V.K. (2004). A simple 
spectrophotometric method for the determination of nicotine in environmental samples. Journal of the Chinese Chemical Society, 51(5A), 949953.

Baliga, S., Muglikar, S., and Kale, R. (2013). Salivary $\mathrm{pH}$ : A diagnostic biomarker. Journal of Indian Society of Periodontology, 17(4), 461465.doi:10.4103/0972-124X.118317

Brandsch, R. (2006). Microbiology and biochemistry of nicotine degradation. Applied microbiology and biotechnology, 69(5), 493-498.

Cppuccino, J. C., Sherman, N., (1992). In: Microbiology: A Laboratory Manual, Addison Wealey Longman Inc, New York, pp. 125-179.

Gurusamy, R., and Natarajan, S. (2013). Current status on biochemistry and molecular biology of microbial degradation of nicotine. The Scientific World Journal, 2013.

Hossain, A. M., and Salehuddin, S. M. (2013). Analytical determination of nicotine in tobacco leaves by gas chromatography-mass spectrometry. Arabian Journal of Chemistry, 6(3), 275-278.

Li, H., Duan, Y., Ma, G., Lei, L., Zhang, K. Q., and Yang, J. (2011). Isolation and characterization of Acinetobacter sp. ND12 capable of degrading nicotine. African Journal of Microbiology Research, 5(11), 1335-1341.

Li, H., Li, X., Duan, Y., Zhang, K. Q., and Yang, J. (2010). Biotransformation of nicotine by microorganism: the case of Pseudomonas spp. Applied microbiology and biotechnology, 86(1), 11-17.

Li, H., Xie, K., Huang, H., and Wang, S. (2014). 6-Hydroxy-3-succinoylpyridine hydroxylase catalyzes a central step of nicotine degradation in Agrobacterium tumefaciens S33. PloS one, 9(7), e103324.

Liu, Y., Wang, L., Huang, K., Wang, W., Nie, X., Jiang, Y. and Tang, H. (2014). Physiological and biochemical characterization of a novel nicotinedegrading bacterium Pseudomonas geniculata N1. PloS one, 9(1), e84399.

McFarland J. (1907). Nephelometer: an instrument for media used for estimating the number of bacteria in suspensions used for calculating the opsonic index and for vaccines. The Journal of the American Medical Association, 14, 1176-1178.

Meng, X. J., Lu, L. L., Gu, G. F., and Xiao, M. (2010). A novel pathway for nicotine degradation by Aspergillus oryzae 112822 isolated from tobacco leaves. Research in microbiology, 161(7), 626-633.

NIDA. (2018, June 6). Cigarettes and Other Tobacco Products. Retrieved from https://www.drugabuse.gov/publications /drugfacts/cigarettes-other-tobaccoproducts.

Niu, T., Chen, C., Ni, J., Wang, B., Fang, Z., Shao, H., and Xu, X. (2000). Nicotine dependence and its familial aggregation in Chinese. International journal of epidemiology, 29(2), 248-252

Qiao, D., Seidler, F. J., and Slotkin, T. A. (2005). Oxidative mechanisms contributing to the developmental neurotoxicity of nicotine and chlorpyrifos. Toxicology and applied pharmacology, 206(1), 17-26.

Singh j., Yadav L., Singh R., Siddiqui I., Singh J. and Srivastava J. (2013). Advanced practical chemistry.

Svendsen, A.B., and Verpoorte, R. (2011). Chromatography of Alkaloids, Part A: Thin-Layer Chromatography. Elsevier.

Wang, S. N., Liu, Z., and Xu, P. (2009). Biodegradation of nicotine by a newly isolated Agrobacterium sp. strain S33. 
Journal of applied microbiology, 107(3), 838-847.

Wei, H., Lei, L., Xia, Z., Liu, S., Liu, P., and Liu, X. (2008). Characterization of a novel aerobic nicotine-biodegrading strain of Pseudomonas putida. Annals of Microbiology, 58(1), 41-45.
Yildiz, D. (2004). Nicotine, its metabolism and an overview of its biological effects. Toxicon, 43(6), 619-632.

Yildiz, D., Ercal, N., and Armstrong, D. W. (1998). Nicotine enantiomers and oxidative stress. Toxicology, 130(2-3), 155-165.

\section{How to cite this article:}

Bhavika Chauhan and Nileshkumar Pandya. 2019. Isolation and Characterization of Nicotine Reducing Probiotics. Int.J.Curr.Microbiol.App.Sci. 8(07): 1193-1200.

doi: https://doi.org/10.20546/ijcmas.2019.807.142 